\title{
Numerical comparison of compound and extracted eye models for high frequency dosimetry
}

\author{
Mario Cvetković(1), Hrvoje Dodig(2), Dragan Poljak ${ }^{(1)}$ \\ (1) Faculty of Electrical Engineering, Mechanical Engineering and Naval Architecture, University of Split, Split, CROATIA \\ e-mail:mcvetkov@fesb.hr,dpoljak@fesb.hr \\ (2) Faculty of Maritime Studies, University of Split, Split, CROATIA \\ e-mail: hdodig@pfst.hr
}

\section{SUMMARY}

This paper compares the numerical results for the induced electric field, the specific absorption rate (SAR), and the corresponding temperature increase in two detailed models of the human eye. The first model features the human eye placed in the free space, while the second one incorporates the eye model in the realistic head model obtained from the magnetic resonance imaging (MRI) scans. The electromagnetic model is based on the hybrid FEM/BEM formulation for the biological tissue, whereas the thermal dosimetry model is based on the bioheat equation solved by using the finite element method. The preliminary analysis showed a similar distribution of the induced electric field along the pupillary axis obtained in both models, however, the numerical results for the SAR and related temperature increase showed discrepancy between the two models, which can be attributed to the high values of induced field in the corneal and scleral regions obtained in the compound eye model.

KEY WORDS: boundary element method; electromagnetic model; finite element method; human eye; hybrid method; thermal dosimetry model.

\section{INTRODUCTION}

The nowadays ubiquitous wireless communication systems have increased the public concern regarding a possible radiation hazard. As the established biological effect of high frequency (HF) electromagnetic (EM) fields is heating of the tissue, the HF exposure assessment is based on the calculation of specific absorption rate (SAR) and related temperature increase in the tissue. This is of particular interest in the case of the exposed human eye and the brain, respectively, since a direct measurement of these quantities in live subjects is rather difficult, if possible at all. 
Hence, the computational methods and their related numerical solutions have become the necessary tools in this exposure assessment [1]-[3]. Computational models used for this particular assessment can be classified as either realistic models of the body (or particular organs) mostly based on magnetic resonance imaging (MRI), e.g. [4], or simplified models, computationally much less demanding but failing to provide accurate results in most of the exposure scenarios [5].

Although the detailed models of the complete human body are readily available, e.g. [6], [7], modelling the complete human body puts a significant burden on the preparation of the computational model and increases the requirements for the computational resources. Moreover, in some cases, especially when the initial assessment is considered, only the particular organ or certain body parts are required.

The choice of when to use the single-organ model or the more complete body model is neither straightforward nor simple. This paper presents a comparison of the results between the single-organ model (termed in this paper as "extracted" or "single-eye model") and the same model of the eye incorporated in the detailed human head model (termed as "compound eye model") exposed to high frequency electromagnetic radiation.

The paper is organised as follows. In the first part, the description of the extracted and the compound eye models, respectively, is given. This is followed by the description of the electromagnetic model based on the hybrid finite element method/boundary element method (FEM/BEM) formulation, and the thermal dosimetry model solved by means of the finite element method [8]. The numerical results for the induced electric field, the specific absorption rate (SAR), and the temperature increase in the two eye models due to the EM plane wave of $1 \mathrm{GHz}$ are presented in the following section. The conclusion is provided in the final part.

\section{THEORETICAL BACKGROUND}

\subsection{EXTRACTED (SINGLE) EYE MODEL}

Human eye is a delicate organ consisting of many fine parts, each performing diverse important functions. Hence, a detailed model of the human eye is very important to account for such small but important subtleties. The cross-section of the human eye displaying its parts is shown in Figure 1.
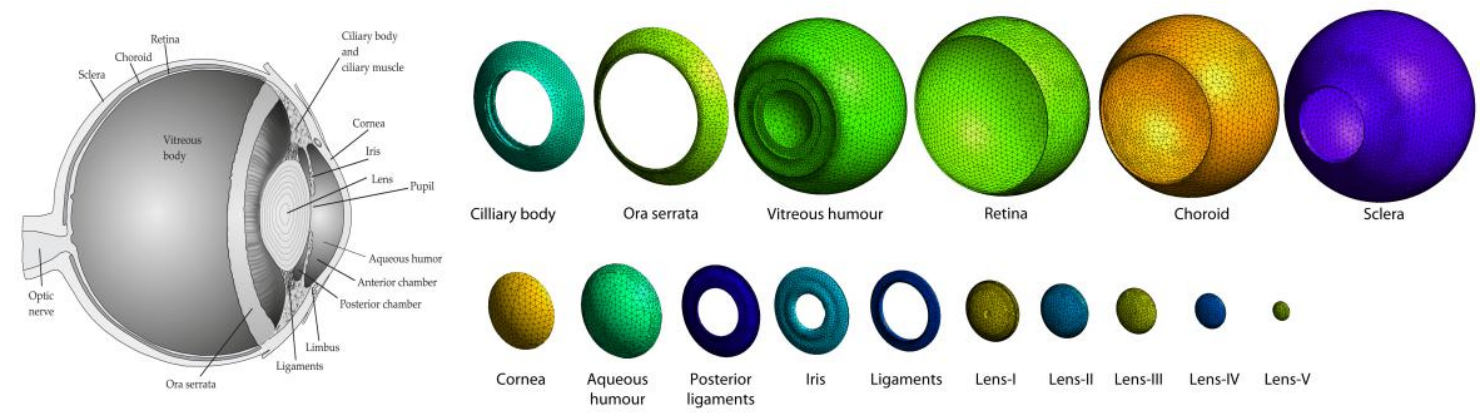

Fig. 1 Cross-section of the human eye (left) and various eye tissues (right) from the compound and the extracted eye model 
Magnetic resonance imaging (MRI) is a radiology technique that can rather accurately capture the details of the human anatomy, however, the spatial resolution of MRI is not accurate enough to capture the fine geometrical details of the human eye. According to some recent studies, ultra-high resolution 7T MRI techniques were able to capture spatial anatomical data with isotropic resolutions of $0.6 \mathrm{~mm}$ and $0.7 \mathrm{~mm}$ [9], whereas [10] reported spatial resolutions of $0.5 \times 0.5 \times 0.6 \mathrm{~mm}^{3}$. To the best of our knowledge, the most accurate MRI scans were performed by using $9.4 T$ magnet [11] achieving voxel volumes of about $0.13 \times 0.13 \times 0.8 \mathrm{~mm}^{3}$.

However, in order to capture the fine details of the eye tissues such as choroid, retina, iris, etc., a much finer resolution is required. It is estimated that in order to have at least several layers of MRI voxels in the retina, we would need spatial MRI resolution of about $30 \times 30 \times 30 \mu^{3}$ or less, otherwise the resulting boundary surfaces would become stair-cased.

Therefore, a detailed geometrical model of the human eye - here referred to as the extracted (single) eye model - has been developed from the available MRI scans, as well as from various medical measurements data. The details of our constructed eye model are reported elsewhere [12].

The extracted eye model consists of 16 various tissues, whose parameters are presented in Table 1 . The frequency-dependent dielectric parameters (electrical conductivity $\sigma$ and relative permittivity $\varepsilon_{r}$ are modelled by using the 4-Cole-Cole method [13]. The last parameter in Table 1 refers to the tissue mass density $\rho$.

Table 1 Tissue parameters used in eye and head models

\begin{tabular}{|c|c|c|c|c|c|c|c|}
\hline Tissue & $\begin{array}{c}\sigma \\
(S / m)\end{array}$ & $\begin{array}{l}\varepsilon \\
(-)\end{array}$ & $\begin{array}{c}\rho \\
(g / m 3)\end{array}$ & $\begin{array}{l}c b \\
(J / \mathrm{kg} / \mathrm{C})\end{array}$ & $\begin{array}{l}\lambda \\
\left(\mathrm{W} / \mathrm{m} /{ }^{\circ} \mathrm{C}\right)\end{array}$ & $\begin{array}{l}W b \\
(W / m 3 / C)\end{array}$ & $\begin{array}{l}Q m \\
(W / m 3)\end{array}$ \\
\hline Brainstem & 0.622 & 38.577 & 1043 & 3600 & 0.503 & 35000 & 10000 \\
\hline Cerebellum & 1.308 & 48.858 & 1039 & 3680 & 0.565 & 35000 & 10000 \\
\hline Head skin & 0.899 & 40.936 & 1050 & 3500 & 0.420 & 9100 & 1000 \\
\hline Liquor & 1.667 & 68.875 & 1035 & 3840 & 0.530 & 0 & 0 \\
\hline Skull & 0.364 & 20.584 & 1900 & 1300 & 0.300 & 1000 & 0 \\
\hline Mandible & 0.364 & 20.584 & 1900 & 1300 & 0.300 & 1000 & 0 \\
\hline Grey Matter & 0.985 & 52.282 & 1039 & 3680 & 0.565 & 35000 & 10000 \\
\hline $\begin{array}{l}\text { Anterior } \\
\text { chamber }\end{array}$ & 1.667 & 68.875 & 1003 & 3900 & 0.580 & 0 & 0 \\
\hline Choroid & 0.729 & 44.561 & 1060 & 3840 & 0.530 & 0 & 0 \\
\hline Ciliary body & 0.978 & 54.811 & 1040 & 3430 & 0.498 & 2700 & 690 \\
\hline Cornea & 1.438 & 54.835 & 1076 & 4200 & 0.580 & 0 & 0 \\
\hline Iris & 0.978 & 54.811 & 1040 & 3430 & 0.498 & 2700 & 690 \\
\hline Ligaments & 0.760 & 45.634 & 1040 & 3300 & 0.420 & 2700 & 690 \\
\hline Ora serrata & 0.882 & 45.711 & 920 & 2500 & 0.250 & 520 & 180 \\
\hline $\begin{array}{l}\text { Posterior } \\
\text { chamber }\end{array}$ & 1.667 & 68.875 & 1000 & 3997 & 0.578 & 0 & 0 \\
\hline Retina & 1.206 & 55.017 & 1039 & 3680 & 0.565 & 35000 & 10000 \\
\hline Sclera & 1.206 & 55.017 & 1076 & 4200 & 0.580 & 13500 & 0 \\
\hline Vitreous body & 1.667 & 68.875 & 1009 & 3997 & 0.594 & 0 & 0 \\
\hline Lens-I & 0.824 & 46.399 & 1100 & 3000 & 0.400 & 0 & 0 \\
\hline Lens-II & 0.824 & 47.011 & 1100 & 3000 & 0.400 & 0 & 0 \\
\hline Lens-III & 0.824 & 47.694 & 1100 & 3000 & 0.400 & 0 & 0 \\
\hline Lens-IV & 0.824 & 48.383 & 1100 & 3000 & 0.400 & 0 & 0 \\
\hline Lens-V & 0.824 & 49.076 & 1100 & 3000 & 0.400 & 0 & 0 \\
\hline
\end{tabular}


The lens is modelled by using five layers (Layers I-V), according to the Gradient Refraction Index (GRIN) model [14], [15].

The boundary surface of the eye model is discretised by using 7.986 triangular elements, while the interior domain of the eye is discretised by using 415.429 tetrahedral elements.

\subsection{COMPOUND EYE MODEL}

The extracted eye model developed in the previous section is included in the model of the human head composed of various head tissues as shown in Figure 2. This model is referred to as the compound eye model.

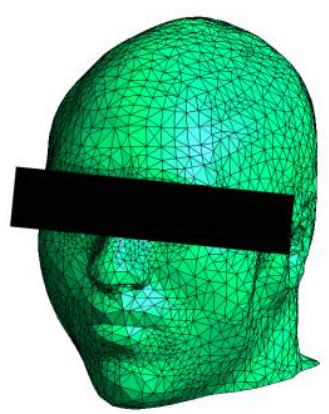

a)

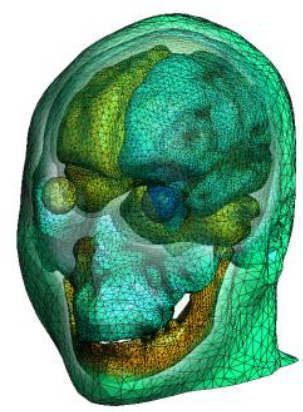

b)

Fig. 2 Model of the human head a), and the overlay depicting various head tissues $b$ ), surrounding the compound eye model

The model of the human head was constructed from the MRI of a 24-year old male [16]. The current implementation of the model is implemented by using 7 tissues in additional to 16 ocular tissues from extracted eye model. The head tissue parameters are also modelled by using the 4-Cole-Cole method [13] and are presented in Table 1 . The boundary surface of the complete head model is discretised by using 5.934 triangular elements, while the head inside is discretised by using 4.073.250 tetrahedral elements.

\subsection{HYBRID FEM/BEM METHOD}

Electromagnetic wave incident on the human eye or head can be treated as the unbounded scattering problem. By using the Stratton-Chu integral expression, the time harmonic electric field in the domain exterior to the head is expressed by the following boundary integral Eq. [8], [17]:

$$
\alpha \vec{E}_{e x t}^{\prime}=\vec{E}_{i n c}^{\prime}+\oint_{\partial V} \hat{n} \times\left(\nabla \times \vec{E}_{e x t}\right) G d S+\oint_{\partial V}\left[\left(\hat{n} \times \vec{E}_{e x t}\right) \times \nabla G+\left(\hat{n} \cdot \vec{E}_{e x t}\right) \nabla G\right] d S
$$

where $\hat{n}$ is an outer normal to surface $\partial V$ bounding the volume $V$ and $\alpha$ is the solid angle subtended at the observation point. $\vec{E}_{\text {ext }}$ and $\vec{E}_{\text {inc }}$ are the total and the incident electric field, respectively, while G denotes the Green's function for the free space given by:

$$
G=G\left(\vec{r}, \vec{r}^{\prime}\right)=\frac{e^{-j k R}}{4 \pi R} ; \quad R=\left|\vec{r}-\vec{r}^{\prime}\right|
$$

where $R$ is the distance from the observation point $\vec{r}$ to the source point $\vec{r}^{\prime}$, and $k$ denotes the wave number. 
Performing some manipulations, (1) can be specified in terms of tangential components of electric field $\vec{E}$ and magnetic field $\vec{H}$ on the boundary surface $\partial V$ :

$$
\alpha \vec{E}_{e x t}^{\prime}=\vec{E}_{i n c}^{\prime}-j \omega \mu \oint_{\partial V} \hat{n} \times \vec{H}_{e x t} G d S+\oint_{\partial V}\left[\left(\hat{n} \times \vec{E}_{e x t}\right) \times \nabla G-\frac{1}{\sigma+j \omega \mu} \nabla_{s} \cdot\left(\hat{n} \times \vec{H}_{e x t}\right) \nabla G\right] d S
$$

Equation (3) is in the proper form pertinent for coupling to the governing differential equations of the interior inhomogeneous domain, given by:

$$
\nabla \times\left(\frac{j}{\omega \mu} \nabla \times \vec{E}_{\text {int }}\right)-(\sigma+j \omega \varepsilon) \vec{E}_{\text {int }}=0
$$

The fields $\vec{E}$ and $\vec{H}$ are approximated by using the edge elements [18] preserving the tangential continuity of the fields on the boundary:

$$
\vec{E}=\sum_{i=1}^{n} \delta_{i} \vec{w}_{i} e_{i}, \quad \vec{H}=\sum_{i=1}^{n} \delta_{i} \vec{w}_{i} h_{i}
$$

The unknown coefficients $e_{i}$ and $h_{i}$, respectively, associated with each edge of the model, are determined from the global system of equations, while the coefficient $\delta_{i}= \pm 1$ is equal to 1 depending on whether the direction of the local edge coincides with the chosen global edge direction.

Vector base function $w_{k}$ is given by [19], $\vec{w}_{k}=N_{i} \nabla N_{j}-N_{j} \nabla N_{i}$, where $N_{i}$ and $N_{j}$ are the first order barycentric shape functions.

After the weighted residual approach is applied to (4), followed by the dot product of (4) with test function $w_{i}$, and by using the Galerkin-Bubnov procedure, it ensues that:

$$
\int_{V} \delta_{i} \vec{w}_{i} \cdot\left[\nabla \times\left(\frac{j}{\omega \mu} \nabla \times \vec{E}_{i n t}\right)-(\sigma+j \omega \varepsilon) \vec{E}_{i n t}\right] d V=0
$$

After applying some standard vector identities, followed by the divergence theorem, the weak form is obtained:

$$
\int_{V}\left[\frac{j}{\omega \mu} \nabla \times \delta_{i} \vec{w}_{i} \cdot \vec{E}_{i n t}-(\sigma+j \omega \varepsilon) \delta_{i} \vec{w}_{i} \cdot \vec{E}_{i n t}\right] d V=\oint_{\partial V} \vec{d} S \cdot \delta_{i} \vec{w}_{i} \times \vec{H}_{i n t}
$$

Now FEM/BEM coupling can be employed by exploiting the fact that the tangential components of electric and magnetic fields must be continuous across the surface $\partial V$. Because of the tangential continuity, it ensues that $\hat{n} \times \vec{E}_{\text {int }}=\hat{n} \times \vec{E}_{\text {ext }}$ and $\hat{n} \times \vec{H}_{\text {int }}=\hat{n} \times \vec{H}_{\text {ext }}$. Thus, it can be written:

$$
\oint_{\partial V} \vec{d} S^{\prime} \cdot \delta_{i} \vec{w}_{i} \times \vec{E}_{i n t}^{\prime}=\oint_{\partial V} \vec{d} S^{\prime} \cdot \delta_{i} \vec{w}_{i} \times \vec{E}_{e x t}^{\prime}
$$

One can now substitute $\vec{E}_{\text {ext }}$ and $\vec{H}_{\text {ext }}$ in (3) with $\vec{E}_{\text {int }}$ and $\vec{H}_{\text {int }}$, respectively, and after inserting (3) into (8), the following double surface integral is obtained:

$$
\begin{aligned}
& \oint_{\partial V} \vec{d} S^{\prime} \cdot \delta_{i} \vec{w}_{i} \times \alpha \vec{E}_{e x t}^{\prime}=\oint_{\partial V} \vec{d} S^{\prime} \cdot \delta_{i} \vec{w}_{i} \times \vec{E}^{\prime} \text { inc }-j \omega \mu \oint_{\partial V} \vec{d} S^{\prime} \cdot \delta_{i} \vec{w}_{i} \times \oint_{\partial V} \hat{n} \times \vec{H}_{i n t} G d S+ \\
& +\oint_{\partial V} \vec{d} S^{\prime} \cdot \delta_{i} \vec{w}_{i} \times \oint_{\partial V}\left(\hat{n} \times \vec{E}_{i n t}\right) \times \nabla G d S-\frac{1}{\omega+j \omega \mu} \oint_{\partial V} \vec{d} S^{\prime} \cdot \delta_{i} \vec{w}_{i} \times \oint_{\partial V} \nabla_{S} \cdot\left(\hat{n} \times \vec{H}_{e x t}\right) \nabla G d S
\end{aligned}
$$


Embedding (5) into (9) results in the system of equations related to the edges at the boundary surface of the problem:

$$
\begin{gathered}
E_{b e m} e_{b e m}=e_{i n c}+H_{b e m} h_{b e m} \\
{\left[E_{f e m}\right] e_{f e m}=\left[H_{f e m}\right] h_{f e m}}
\end{gathered}
$$

where $e_{b e m}$ and $h_{b e m}$ are unknown coefficients associated with the boundary surface of the scattering problem, $e_{i n c}$ is the known coefficient calculated from the incident field, and matrices $E_{b e m}$ and $H_{b e m}$ arise from the boundary integral Eq. (9), while matrices $\left[E_{f e m}\right]$ and $\left[H_{f e m}\right]$ stem from the FEM Eq. (7).

\subsection{VERIFICATION OF ELECTROMAGNETIC MODEL}

The electromagnetic model based on the hybrid formulation is verified by comparing it to the Mie series solution of the radar cross-section (RCS) computations for the coated metallic sphere at the interior resonance frequency. The validity of this approach was also shown in the previous research [8], [17], [20], [21], whereas it is repeated here for the reader's convenience.

This verification is of particular interest due to the following: a) the material properties change inside the sphere, and b) some research papers show significant errors at the interior resonance frequency [22], [23].

The RCS of the coated metallic sphere at $300 \mathrm{MHz}$, corresponding to the interior resonance frequency, is shown in Figure 3. The comparison of the FEM/BEM results with the analytical solution, obtained by using the Mie theory [23], showed excellent agreement, as shown in Figure 3.
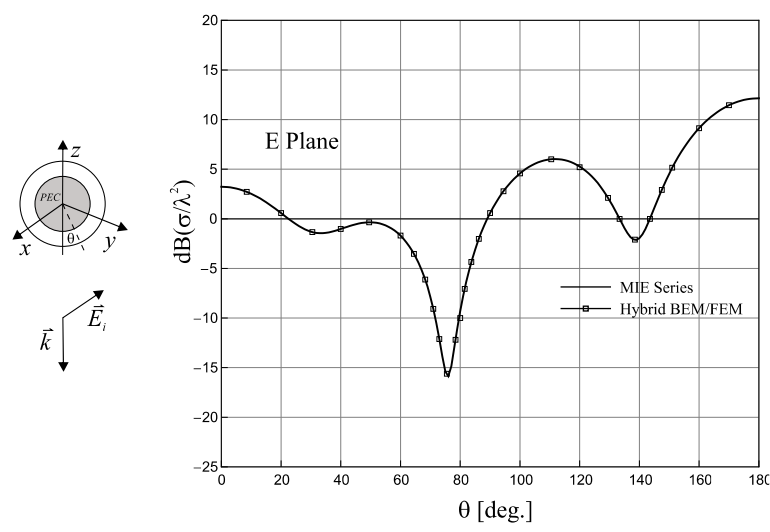

Fig. 3 Bistatic RCS of a metallic sphere coated with dielectric layer of $\varepsilon_{r}=4$ at the resonant frequency of 300 MHz. Comparison of hybrid FEM/BEM formulation and analytical Mie theory based solution from [23]

\subsection{THERMAL DOSIMETRY MODEL}

The temperature distribution in the human eye can be determined numerically by using the stationary form of the Pennes' bioheat equation [24]:

$$
\nabla \cdot \lambda \nabla T+W_{b} c_{b} T_{a}-T+Q_{m}+Q_{e x t}=0
$$


where the heat generated due to metabolic processes is given by $Q_{m}, W_{b}$ and $c_{b}$ refer to the volumetric perfusion rate and the specific heat capacity of blood, respectively, $\lambda$ is the thermal conductivity of the tissue, and $T_{a}$ is the arterial blood temperature. Thermal parameters of the eye and human head tissues are given in Table 1. The last term in (12) is the heat generated from the absorbed energy due to the EM wave in the biological tissue, and can be calculated from:

$$
Q_{\text {ext }}=\rho \cdot S A R
$$

where $\rho$ is the tissue density given in Table 1 . The specific absorption rate (SAR) is readily found by using:

$$
S A R=\frac{\sigma}{2 \rho}|\vec{E}|^{2}
$$

where $\sigma$ is the tissue electrical conductivity and $\vec{E}$ is the induced electric field obtained by using the electromagnetic model.

In case when no incident EM field is present, the term $Q_{e x t}$ must be omitted from (12).

Equation (12) is supplemented by the Neumann boundary condition imposed on the boundary surface of the model:

$$
\lambda \frac{\partial T}{\partial \vec{n}}=-h_{c}\left(T-T_{a}\right)
$$

where $h_{c}$ is the convection coefficient between the surface and the surroundings. For the interface between the eye and air we have implemented $h_{c}=20 \mathrm{~W} / \mathrm{m}^{2}{ }^{\circ} \mathrm{C}$, whereas for the interface between the eye and the surrounding tissue $h_{c}=40 \mathrm{~W} / \mathrm{m}^{2}{ }^{\circ} \mathrm{C}$.

The forced convection, and the heat loss due to radiation, respectively, can be neglected. The bioheat Eq. (12) can be solved by using the finite element method (FEM) based on the weighted residual approach. The approximate solution of (12) is expanded in terms of the known basis functions and the unknown coefficients. After multiplying (12) by a set of weighting functions and integrating over the domain, after some work, the suitable expression for the finite element method is obtained:

$$
\begin{aligned}
& \int_{\Omega} \lambda \nabla T \cdot \nabla W_{j} d \Omega+\int_{\Omega} W_{b} T W_{j} d \Omega+\int_{\partial \Omega} h_{s} T W_{j} d S= \\
& =\int_{\Omega}\left(W_{b} T_{a}+Q_{m}+Q_{e x t}\right) W_{j} d \Omega+\int_{\partial \Omega} h_{s} T_{a m b} W_{j} d S
\end{aligned}
$$

Implementing the Galerkin-Bubnov procedure, followed by the standard finite element discretisation of (16), the weak formulation for the finite element domain $\Omega_{e}$ leads to the matrix:

$$
K^{e} T^{e}=M^{e}+P^{e}
$$

where $K^{e}$ is the finite element matrix:

$$
K_{j i}^{e}=\int_{\Omega_{e}} \lambda^{e} \nabla W_{i} \cdot \nabla W_{j} d \Omega_{e}+\int_{\Omega_{e}} W_{b}^{e} W_{i} W_{j} d \Omega_{e},
$$

$M^{e}$ is the flux vector on the boundary $\partial \Omega_{e}$ of the finite element: 


$$
M_{j}^{e}=\int_{\Omega_{\Omega}} \lambda_{e}^{e} \frac{\partial T}{\partial \hat{n}} W_{j} d S_{e}
$$

and $P^{e}$ is the finite element source vector:

$$
P_{j}^{e}=\int_{\Omega_{e}}\left(W_{b}^{e} T_{a}+Q_{m}^{e}+Q_{e x t}^{e}\right) W_{j} d \Omega_{e},
$$

respectively.

By solving (18)-(20) for each $N$ element, the global matrix is assembled from the contribution of the local finite element matrices, while the global flux and the source vectors are assembled from the local flux and the local source vectors, respectively:

$$
K \quad T=M+P .
$$

The solution to (21) represents the temperature at the tetrahedral nodes used to discretise the problem domain.

\section{NUMERICAL RESULTS}

The numerical results obtained by using the hybrid FEM/BEM formulation for the electric field, the specific absorption rate (SAR) induced in the extracted and the compound model of the eye, and the corresponding temperature increase, respectively, are given in Figures 4-10. The incident plane wave of $1 \mathrm{GHz}$ frequency is vertically polarised and directed towards the corneal surface, perpendicular to the coronal head/eye cross-section.

\subsection{ELECTRIC FIELD}

Figure 4 shows the results for the induced field on the transverse cross-sections of the extracted eye model, and the compound eye model, respectively, due to $1 \mathrm{GHz}$ vertically polarised EM wave.

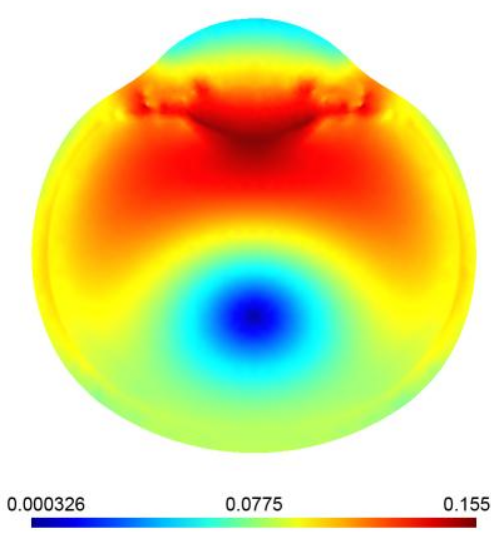

a)

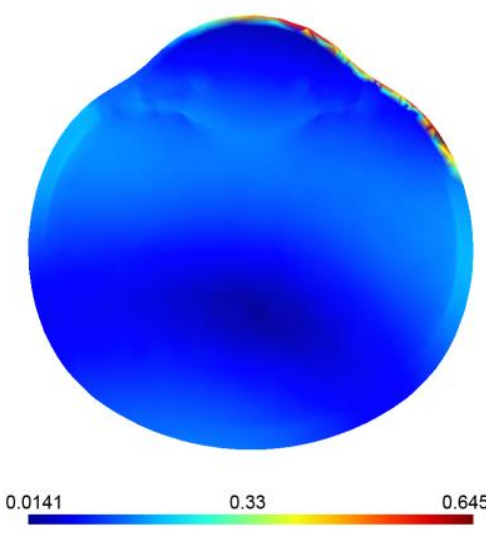

b)

Fig. 4 Induced electric field due to $1 \mathrm{GHz}$ vertically polarised EM wave in the transverse cross-section of the: a) Extracted eye model, b) Compound eye model

Details on the electric field distribution along the eye visual axis are illustrated in Figure 5, where the comparison between two models is given. Figure 5 shows a similar distribution of 
the induced electric field along the pupillary axis, although this may not be obvious from Figure 4.

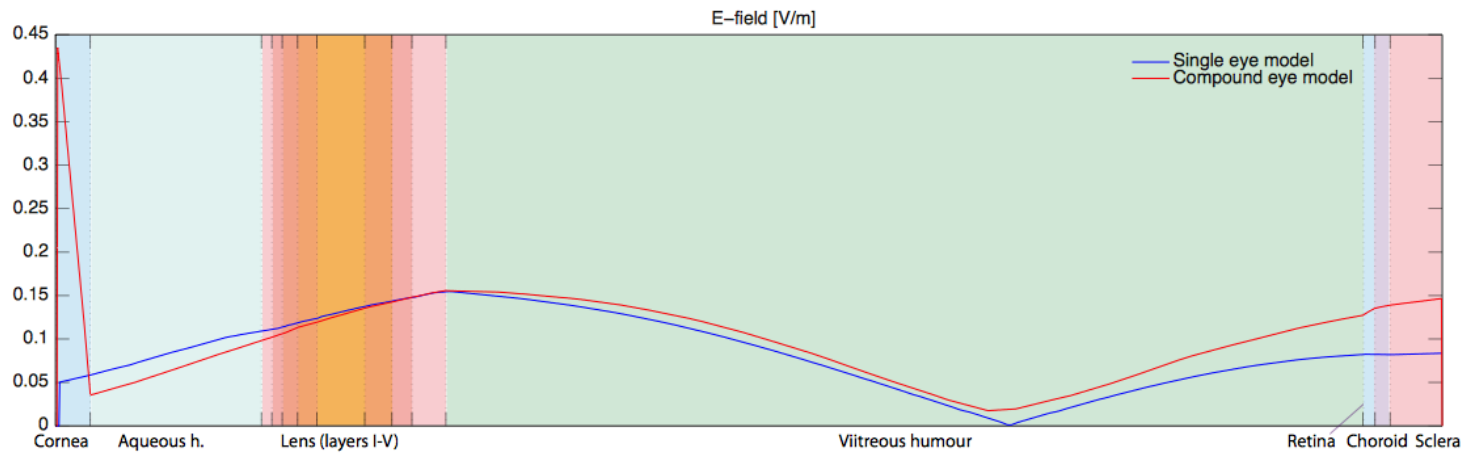

Fig. 5 Comparison of the induced electric field along the visual axis of the extracted eye model (blue line) and the compound eye model (red line)

Also, the results from Figure 4 show that the higher values of the induced electric field in the compound eye model, which are incorporated in the whole head model, are obtained in the superficial region of the eye, namely corneal and scleral region. More details on the electric field distribution on the surface of both eye models can be found in Figure 6.

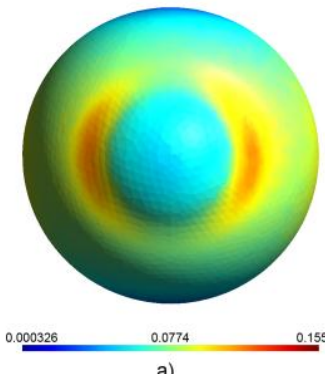

a)

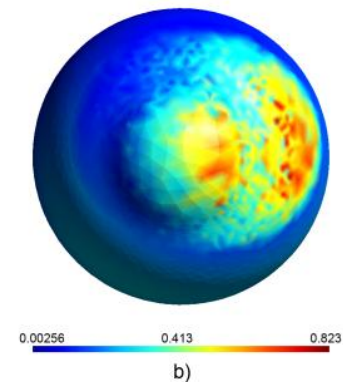

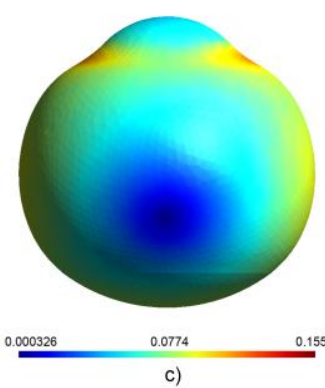

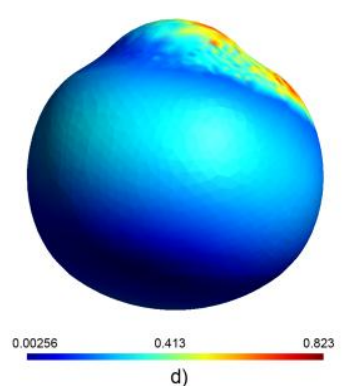

Fig. 6 Induced electric field due to $1 \mathrm{GHz}$ vertically polarised EM wave on the surface of the eye. Anterior view a) and b), and top view c) and d), of extracted and compound eye model, respectively

\subsection{SPECIFIC ABSORPTION RATE}

The corresponding SAR results are shown in Figures 7 and 8. Figure 7 shows the results for the computed SAR on the transverse cross-sections of the extracted eye model, and the compound eye model, respectively. Again, higher values for the SAR are obtained in the compound eye model in the right part of corneal and scleral regions. 


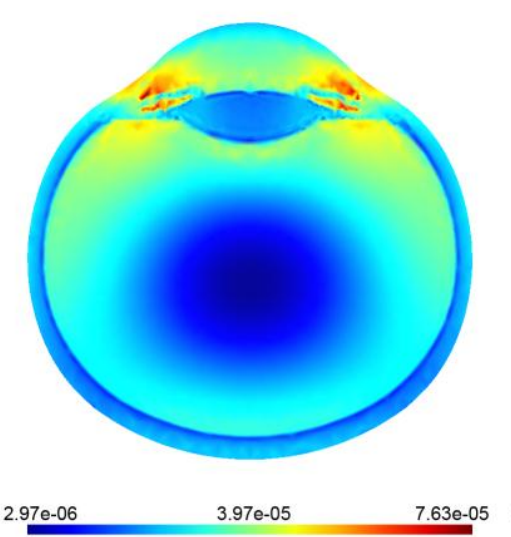

a)

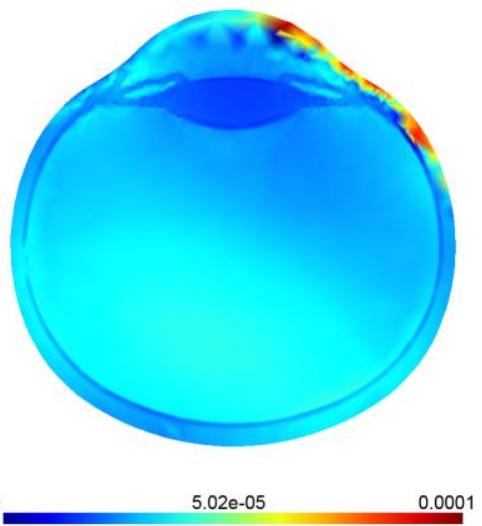

b)

Fig. 7 Induced SAR due to $1 \mathrm{GHz}$ vertically polarised EM wave in the transverse cross-section of the:

a) extracted eye model, b) compound eye model

This non-symmetric distribution of SAR in the compound eye model, contrary to the symmetrical distribution obtained in the extracted eye model, will result in a somewhat different distribution of SAR along the eye pupillary axis, as shown in Figure 8.

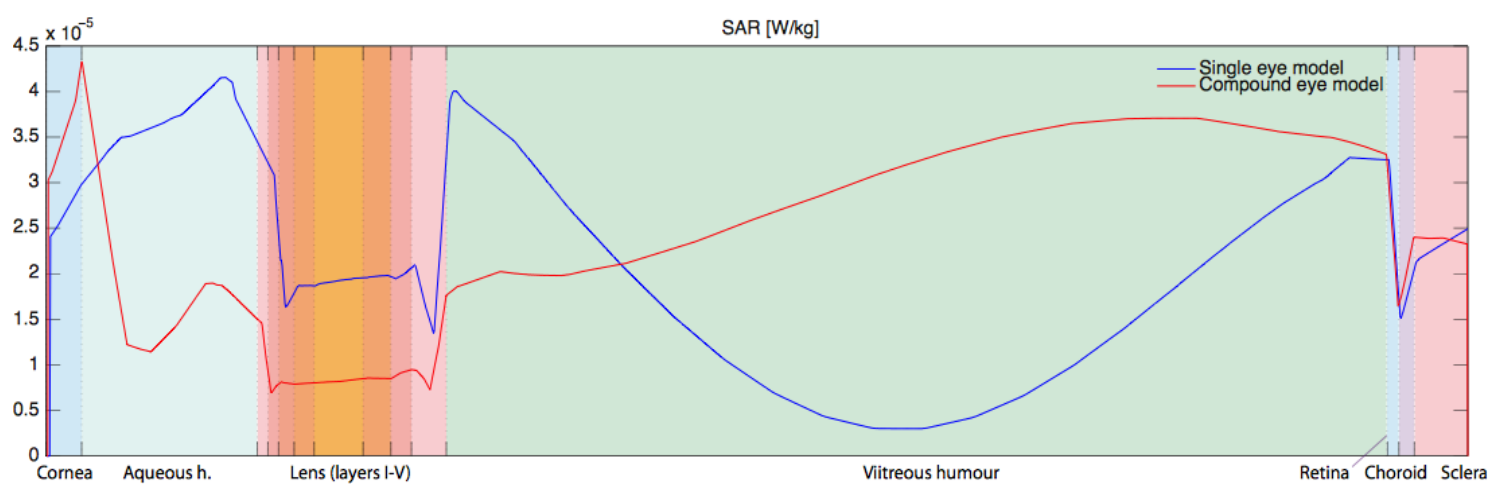

Fig. 8 Comparison of the induced SAR along the visual axis of the extracted eye model (blue line) and the compound eye model (red line)

\subsection{TEMPERATURE DISTRIBUTION}

The final set of results is related to the temperature distribution in two eye models. Figures 9 and 10 show the results of the steady-state temperature in the eye, i.e. when no EM wave is incident, and the results of the corresponding temperature increase due to absorbed EM energy.

Figure 9 shows the results on the horizontal axial slice of both models. A very similar temperature gradient is discernible from the cornea to the sclera in the steady-state case, although different maximum values are obtained. As the compound eye model is surrounded with other head tissues, the maximum temperature obtained in the scleral region is close to the body temperature. The extracted eye model, on the other hand, is surrounded with air set to an ambient temperature of $22^{\circ} \mathrm{C}$, resulting in a considerably lower maximum temperature. 

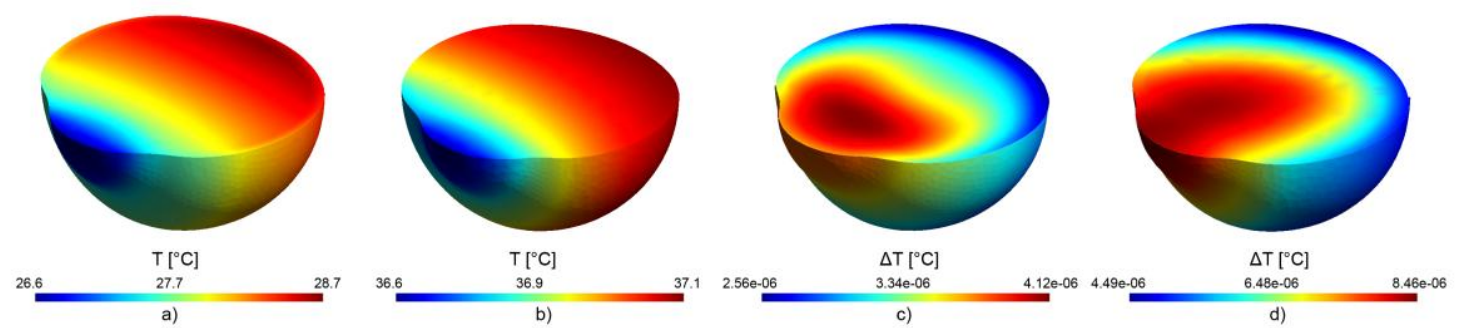

Fig. 9 Horizontal axial slice of the steady-state temperature distribution in a) extracted model, b) compound model, and the temperature increase in c) extracted model, d) compound model

The comparison of the steady-state temperature distribution along the visual axis of the extracted eye and the compound eye, respectively, can be seen in Figure 10.

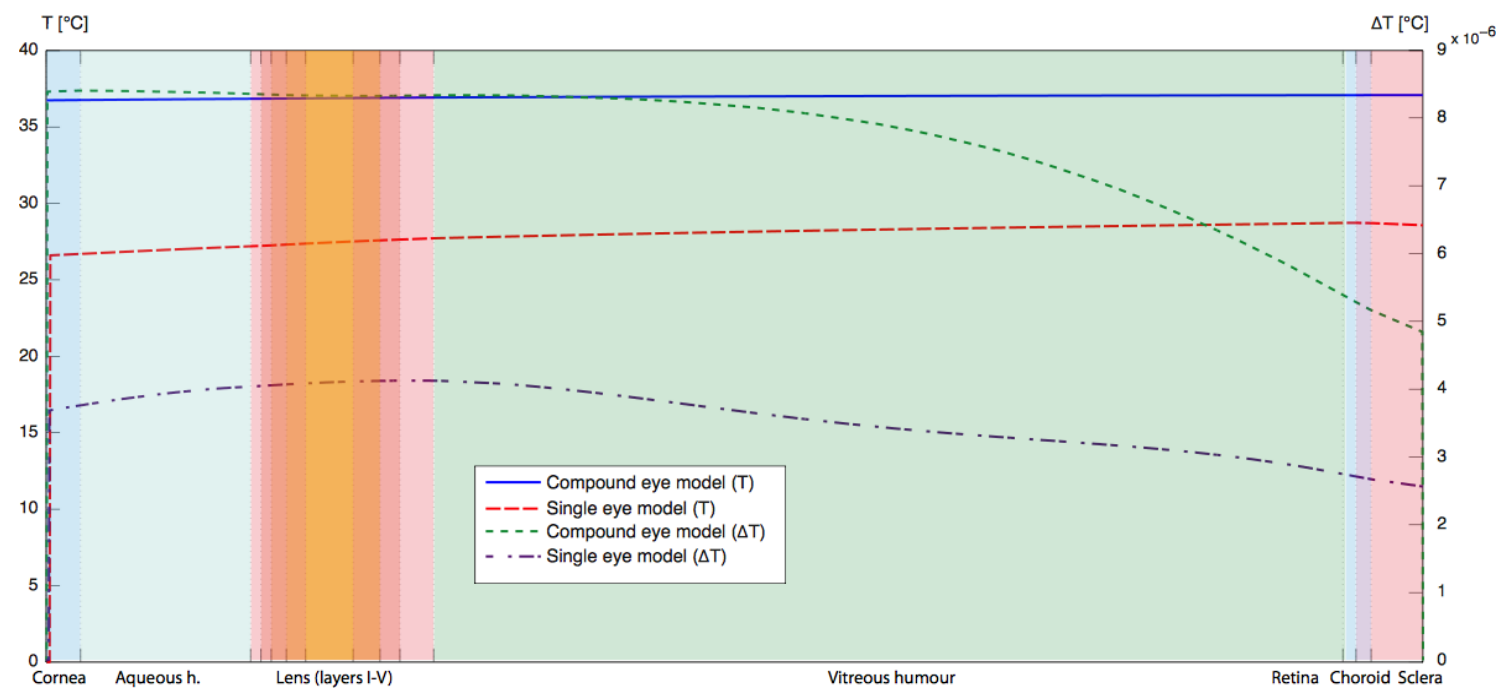

Fig. 10 Comparison of the steady-state temperature distribution and the temperature increase along the visual axis of the extracted eye model and the compound eye model

The results of temperature increase when the eye is exposed to the incident plane EM wave are presented in Figures 9c) and d) and Figure 10. Although both models obtained similar temperature gradients along the eye axis, as shown in Figure 10, the compound eye model obtained more spread of temperature hot-spots in the horizontal direction along the axis of incidence. In the extracted eye model, the hot-spots formed in eye lens, whereas in the compound eye model the hot-spots formed in the cornea and lens region spreading also further to the vitreous region.

\section{CONCLUSION}

This paper presents the comparison of the induced electric field, the specific absorption rate, and the corresponding temperature increase in the extracted or the single eye model and the compound eye model incorporated in the detailed human head exposed to the high frequency electromagnetic radiation. The numerical results obtained by using the hybrid FEM/BEM formulation showed similar distributions of the induced electric field along the pupillary axis in both eye models. Although some discrepancy between the SAR distribution along the same axis was showed in the results for the two models, in general, similar values were reported, 
suggesting the usefulness of the extracted eye model in the initial assessment of the EM plane wave exposure. The thermal dosimetry model showed similar gradients of the eye temperature field for the steady-state case, although the compound eye model obtained higher values of the maximum temperature in the sclera due to the surrounding head tissue and the employed boundary conditions. The temperature distribution in the case of high frequency exposure, on the other hand, showed hot-spots forming in different tissues of extracted and compound models, respectively, suggesting that the extracted eye model should be used carefully when considering realistic thermal dosimetry scenario.

\section{REFERENCES}

[1] J. Hand, Modelling the interaction of electromagnetic fields (10 MHz-10 GHz) with the human body: methods and applications, Physics in medicine and biology, Vol. 53, No. 16, p. R243, 2008. DOI: $10.1088 / 0031-9155 / 53 / 16 / \mathrm{r} 01$

[2] D. Poljak, Electromagnetic fields: Environmental exposure, in Encyclopedia of environmental health, Elsevier, 2011. DOI: 10.1016/b978-0-444-52272-6.00422-0

[3] D. Poljak, M. Cvetković, H. Dodig and A. Peratta, Electromagnetic-thermal analysis for human exposure to high frequency HF radiation, International Journal of Design \& Nature and Ecodynamics, Vol. 12, No. 1, pp. 55-67, 2017.

DOI: $10.2495 /$ dne-v12-n1-55-67

[4] K.D. Singh, N.S. Logan and B. Gilmartin, Three-dimensional modelling of the human eye based on magnetic resonance imaging, Investigative ophthalmology \& visual science, Vol. 47, No. 6, pp. 2272- 2279, 2006. DOI: $10.1167 /$ iovs.05-0856

[5] D. Poljak, Human Exposure To Electromagnetic Fields, WIT Press, Southampton-Boston, 2003.

[6] M.J. Ackerman, The visible human project, Proceedings of the IEEE, Vol. 86, No. 3, pp. 504-511, 1998. DOI: $\underline{10.1109 / 5.662875}$

[7] J.W. Massey and A.E. Yilmaz, AustinMan and AustinWoman: High-fidelity, anatomical voxel models developed from the VHP color images, in $201638^{\text {th }}$ Annual International Conference of the IEEE Engineering in Medicine and Biology Society (EMBC), pp. 33463349, 2016. DOI: 10.1109/EMBC.2016.7591444

[8] H. Dodig, D. Poljak and A. Peratta, Hybrid BEM/FEM edge element computation of the thermal rise in the 3D model of the human eye induced by high frequency EM waves, in Software, Telecommunications and Computer Networks (SoftCOM), 2012 20th International Conference on IEEE, pp. 1-5, 2012.

[9] B.U. Forstmann, M.C. Keuken, A. Schafer, P.-L. Bazin, A. Alkemade and R. Turner, Multimodal ultra-high resolution structural 7T MRI data repository, Scientific data, Vol. 1, 2014. DOI: $\underline{10.1038 / \text { sdata.2014.50 }}$

[10] M.C. Keuken, P.L. Bazin, A. Schafer, J. Neumann, R. Turner and B.U. Forstmann, Ultra-high 7T MRI of structural age-related changes of the subthalamic nucleus, Journal of Neuroscience, Vol. 33, No. 11, pp. 4896-4900, 2013.

DOI: 10.1523/ineurosci.3241-12.2013 
[11] J. Budde, G. Shajan, K. Scheffler and R. Pohmann, Ultra-high resolution imaging of the human brain using acquisition-weighted imaging at $9.4 \mathrm{t}$, Neuroimage, Vol. 86, pp. 592598, 2014. DOI: $\underline{10.1016 / j . n e u r o i m a g e .2013 .08 .013}$

[12] H. Dodig, M. Cvetković and D. Poljak, Detailed human eye GRIN-based model for the electromagnetic-thermal dosimetry, in preparation.

[13] C. Gabriel, Compilation of the dielectric properties of body tissues at RF and microwave frequencies, Brooks Air Force Base, TX. Report: AL/OE-TR-1996-0037, Tech. Rep., 1996. DOI: $10.21236 /$ ada303903

[14] A. Gullstrand, The optical system of the eye, Physiological Optics, Vol. 1, pp. 350-358, 1909.

[15] J.A. Diaz, C. Pizarro and J. Arasa, Single dispersive gradient-indexprofile for the aging human lens, Journal of the Optical Society of America, Vol. 25, No. 1, pp. 250-261, 2008. DOI: $10.1364 /$ josaa. 25.000250

[16] I. Laakso, S. Tanaka, S. Koyama, V. De Santis and A. Hirata, Inter-subject variability in electric fields of motor cortical tDCS, Brain Stimulation, Vol. 8, No. 5, pp. 906-913, 2015. DOI: $10.1016 /$ j.brs.2015.05.002

[17] D. Poljak, D. Cavka, H. Dodig, C. Peratta and A. Peratta, On the use of the boundary element analysis in bioelectromagnetics, Engineering Analysis With Boundary Elements, Vol. 49, pp. 2-14, 2014. DOI: 10.1016/i.enganabound.2014.02.008

[18] J.-C. Nedelec, Mixed finite elements in R3, Numerische Mathematik, Vol. 35, No. 3, pp. 315-341, 1980.

[19] J.L. Volakis, A. Chatterjee and L.C. Kempel, Finite Element Method Electromagnetics: Antennas, Microwave Circuits, and Scattering Applications, John Wiley \& Sons, Vol. 6 1998. DOI: $10.1109 / 9780470544655$

[20] H. Dodig, S. Lallchere, P. Bonnet, D. Poljak and K. El Khamlichi Drissi, Stochastic sensitivity of the electromagnetic distributions inside a human eye modeled with a 3D hybrid BEM/FEM edge element method, Engineering Analysis with Boundary Elements, Vol. 49, pp. 48-62, 2014. DOI: 10.1016/j.enganabound.2014.04.005

[21] D. Poljak, M. Cvetković, A. Peratta, C. Peratta, H. Dodig and A. Hirata, On some integral approaches in electromagnetic dosimetry, in BioEM 2016, Ghent, Belgium, 5-10 June 2016, pp. 289-296, 2016.

[22] A.F. Peterson, The "interior resonance" problem associated with surface integral equations of electromagnetics: Numerical consequences and a survey of remedies, Electromagnetics, Vol. 10, No. 3, pp. 293-312, 1990.

DOI: $\underline{10.1080 / 02726349008908245}$

[23] Y. Ji, H. Wang and T.H. Hubing, A numerical investigation of interior resonances in the hybrid FEM/MoM method, IEEE Transactions on Antennas and Propagation, Vol. 51, No. 2, pp. 347-349, 2003. DOI: 10.1109/tap.2003.809084

[24] H.H. Pennes, Analysis of tissue and arterial blood temperatures in the resting human forearm. 1948, Journal of Applied Physiology, Vol. 85, No. 1, pp. 5-34, 1998.

DOI: $\underline{10.1152 / j a p p l .1998 .85 .1 .5}$ 